\title{
Trivium
}

Revue franco-allemande de sciences humaines et sociales - Deutsch-französische Zeitschrift für Geistesund Sozialwissenschaften

$10 \mid 2012$

Lisibilité

\section{Im Laboratorium der Schrift-Figur}

\section{Louis Marin}

Traducteur : Regine Herrmannsdörfer

\section{Q OpenEdition}

\section{Journals}

\section{Édition électronique}

URL : http://journals.openedition.org/trivium/4217

DOI : $10.4000 /$ trivium.4217

ISSN : 1963-1820

\section{Éditeur}

Les éditions de la Maison des sciences de l'Homme

\section{Référence électronique}

Louis Marin, «Im Laboratorium der Schrift-Figur », Trivium [Online], 10 | 2012, online erschienen am 30 März 2012, abgerufen am 08 September 2020. URL : http://journals.openedition.org/trivium/4217

DOI : https://doi.org/10.4000/trivium.4217

Ce document a été généré automatiquement le 8 septembre 2020

\section{(c) (i) () $\Theta$}

Les contenus des la revue Trivium sont mis à disposition selon les termes de la Licence Creative Commons Attribution - Pas d'Utilisation Commerciale - Pas de Modification 4.0 International. 


\title{
Im Laboratorium der Schrift-Figur
}

\author{
Louis Marin \\ Traduction : Regine Herrmannsdörfer
}

\section{NOTE DE L'ÉDITEUR}

Wir danken Françoise Marin für die freundliche Genehmigung, diesen Artikel zu übersetzen. Der französische Originaltext, erhältlich als PDF-Datei auf der Louis Marin gewidmeten Website, enthält eine Reihe Abbildungen, die wir an dieser Stelle leider nicht in ihrer Gesamtheit reproduzieren können.

Ut Pictura Poesis erit; similisque Poesi Sit Pictura; refert par aemula quaeque sororem Alternantque vices et nomina; muta Poesis Dicitur haec, Pictura loquens solet illa vocari. ${ }^{1}$ Charles Du Fresnoy, De Arte Graphica, 1667, V. 1-4.

Picassos dichterisches Euvre, inzwischen durch eine neuerliche Ausstellung ${ }^{2}$ und eine großartige Edition ${ }^{3} \mathrm{zu}$ größerer Bekanntheit und Anerkennung gelangt, bietet die ausgezeichnete Gelegenheit für einen Einstieg in den modernen und zeitgenössischen Kommentar des alten Horazischen Diktums ut pictura poesis. Im Laufe der letzten dreißig Jahre zeigte sich dieser Kommentar in unterschiedlichen Formen. Sie reichen von umfänglichen Studien »strukturaler« Poetik, wie Jakobsons Artikel über Blake, Rousseau und Klee ${ }^{4}$, bis zu jüngeren Arbeiten aus dem Feld der Rhetorikgeschichte, zum Teil als Antwort auf die strukturale Analyse konzipiert, wenn nicht gar als Rückschritt hinter diese. ${ }^{5}$ So hat man uns etwa eine französische Übersetzung von R. W. Lees Studie »Ut pictura poesis « angekündigt, die 1942 (!) in Art Bulletin ${ }^{6}$ erschien. Es ist dies wohl weniger ein Zeichen für den üblichen und unverzeihlichen nationalen Rückstand in Sachen Publikationen, als vielmehr für eine heutige "Fin de Siècle«Rückwendung zu diversen Theorien und Methoden vergangener Zeiten.

Diesseits oder jenseits dieser zeitgenössischen Befunde zur Geschichtswissenschaft und Epistemologie bietet Picassos poetisches CEuvre gleicherweise die Gelegenheit, um 
erneut einer Frage nachzugehen, die Vers 361 des Horazischen Ars poetica ebenfalls aufwerfen könnte, der Frage nämlich nach den Bezügen von Text und Bild, der man sich vor mehr als zwanzig Jahren widmete und mit der man sich hier und dort noch immer beschäftigt. Eine Gelegenheit also, um diesen Bereich der Forschung noch einmal zu erkunden.

Im Rahmen der Semiologie des Visuellen, einer damals noch jungen, äußerst ambitionierten Wissenschaft, die ihre Ansätze selbstbewusst vertrat, da sie zunächst unter der Ägide der Saussureschen Linguistik stand, fragte man, ob es möglich sei, das Bild als einen Text zu untersuchen; wobei die umgekehrte Fragestellung - den Text als ein Bild $\mathrm{zu}$ untersuchen - nicht von gleicher theoretischer und methodischer Dringlichkeit zu sein schien. "Lesen Sie die Geschichte und das Gemälde«, schrieb Poussin aus Rom an Chantelou, als er ihm 1637 Die Mannalese sandte. Nimmt man nun Poussins Weisung als Devise der damaligen Forschung, so ist sie in doppelter Hinsicht $\mathrm{zu}$ verstehen. Zum einen ging es darum, die Beziehungen zwischen einem Text und einem Gemälde zu studieren: zwischen einem voraufgehenden Text, z. B. im Falle von Poussin einer Stelle aus Exodus, und dem Werk, das diese Episode illustrieren sollte. Über die bloße direkte ikonographische Recherche hinaus fragte man damals, wie, durch welche allgemeinen und besonderen Mittel eine Erzählung zu einem Bild würde, welchen spezifischen Erfordernissen der Maler Rechnung zu tragen habe, sowohl in Bezug auf das pikturale Medium und die visuelle Substanz als auch in Bezug auf die visuellen Wahrnehmungs- und Betrachtungsarten des Werkes, um gleichwohl die Geschichte, die den Gegenstand seines Werkes bildet, »visuell« zu erzählen; welchen, aus den allgemeingültigstenKategorisierungen von Raum, Zeit und deren Repräsentation resultierenden Zwängen er unterliege, die in ebenjenem geschichtlichen Moment und an jenem kulturellen Ort alsGesetze und Normendie Schöpfertätigkeit des Malers ebenso wie das Schauen des Betrachters des Werkes bestimmen.

Somit durfte sich die Untersuchung der Beziehungen zwischen Text und Bild nicht allein auf den Schritt der »Illustrierung« des Textes durch das Bild beschränken, wie komplex diese Prozesse, von mir allzu schnell Illustrierung genannt, auch sein mochten. Es stellte sich die ebenso unabweisliche Frage, welchen Text das Bild entstehen ließ, sofern man das Bild wie einen Text lesen konnte, und welche differentiellen Bedingungen es hierbei gab. Ließ sich dem Bild ein Diskurs entlocken, der ein Diskurs des Bildes wäre und kein Diskurs über das Bild? Wenn man Poussins Brief an Chantelou aufmerksam las, stellte man fest, dass der Meister eine solche »Lektüre« für möglich hielt:

"Lesen Sie die Geschichte und das Gemälde, um zu erkennen, ob jedes Ding dem Gegenstand gemäß ist."

Und er ergänzte (worin ich mir erlaube einen Hinweis auf so etwas wie ein generatives Modell sowohl der Komposition als auch der Lektüre zu sehen):

»Die sieben ersten Figuren linker Hand werden Ihnen alles sagen, was ich hier

schrieb, und alles Übrige ist vom selben Stoff.«

Kurz, es wollte eine umfassende Problematik der Beschreibung und Interpretation von Kunstwerken erarbeitet sein, mit der sich die doppelte Klippe umschiffen ließ einerseits der »Unsagbarkeit« der Malerei, die schlimmstenfalls Geschwätzigkeit produziert und bestenfalls dazu führt, dass man über Bildeindrücke spricht; andererseits der Verwendung geborgter linguistischer Modelle, die nie für die Malerei 
gedacht gewesen waren: eine Problematik des Textes des Bildes, des Diskurses des Gemäldes, die Konzepte entwickeln sollte, um beides möglich und operativ zu machen.

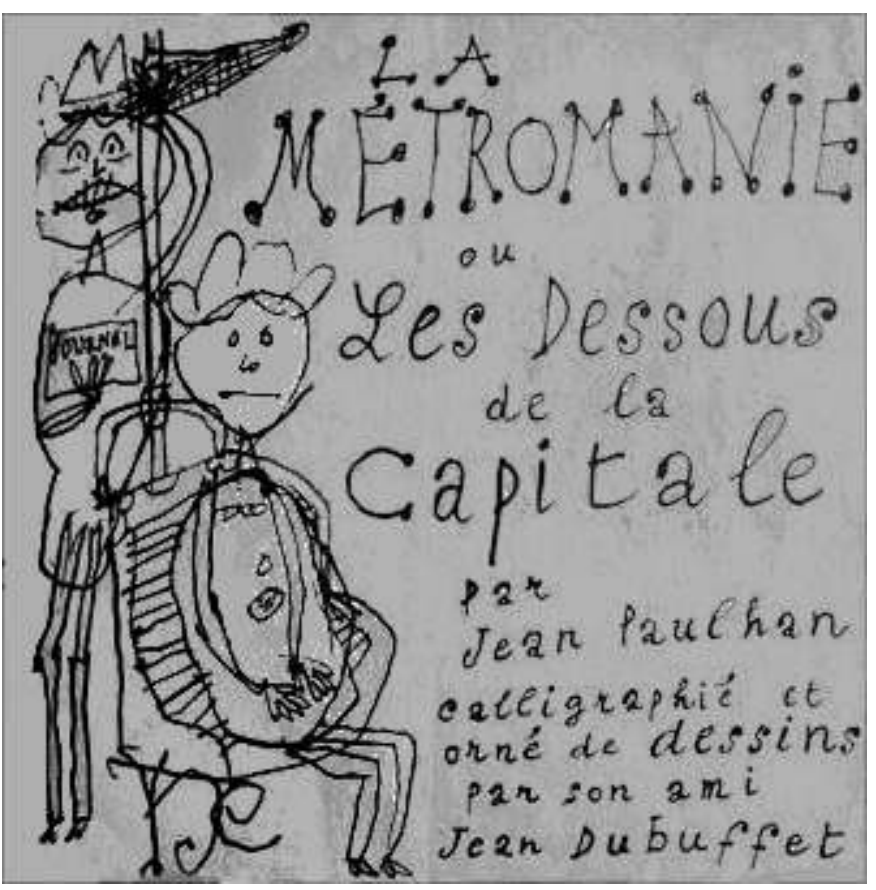

Jean Dubuffet, La Métromanie mit Widmung an Jean Paulhan, 1949 (c) ADAGP, Paris 2012

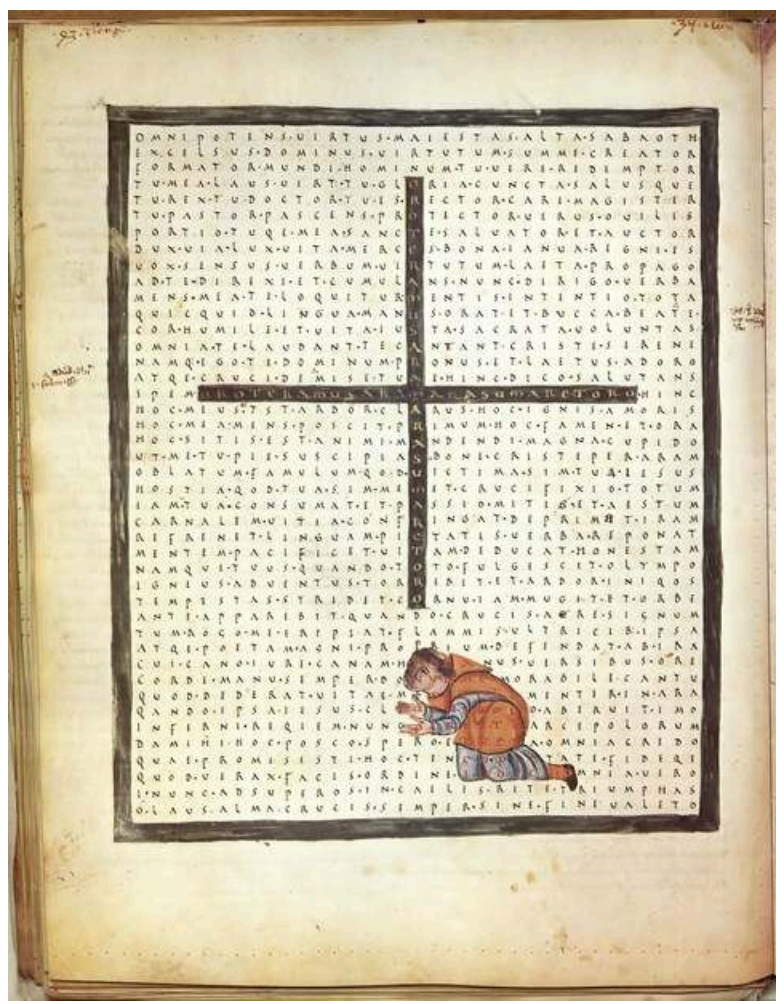

Hrabanus Maurus, Anbetung des Kreuzes, aus: De Laudibus sanctae crucis, Gedichtsammlung, 815

"Lesen Sie die Geschichte und das Gemälde«, Poussins Weisung meinte zunächst die Beziehungen von Text und Bild. Doch weiterhin verlangte sie, den Text im Bild oder Gemälde zu untersuchen und umgekehrt, das Bild (oder Gemälde) im Text, jene 
Verflechtungen also von Text und Bild, in denen der Text Gewebe wird, mit und im Bild, in denen er es textualisiert, in denen das Bild Ikon wird, mit und im Text, in denen das Bild den Text ikonisiert. Beispielhaft dafür: Inschriften, Bildlegenden, Signaturen, Lettern, Markierungen oder Zeichen, die sich in je spezifischer Weise mit den Figuren, Formen, Strichen, Farbflecken in Beziehung setzen, sei es in einem Bild, einem Gemälde, einem Stich oder einer Zeichnung. Oder umgekehrt, Bilder, Illustrationen, Karten, Pläne, Diagramme, Skizzen und sonstige Formen, die am Rand einer Seite, in ihrer Mitte oder auf ihrer Rückseite den handgeschriebenen oder gedruckten Text bearbeiten und ihn arbeiten lassen, sei es im Gedächtnis oder in der Phantasie des Schriftstellers, und dies vielleicht unterhalb seiner Bewusstseinsschwelle; aber auch Frontispize und Vignetten am Anfang, innerhalb oder am Ende eines Buches, die sowohl figurative Zeichen seiner Gliederungen sind als auch Bilder, die im geschriebenen Text jenseits oder diesseits der Interpunktion eigene Akzente setzen; Bilder, die dem zum Text gehörenden Leerraum zwischen Buchstaben und Zeilen eine gewisse Gestalt geben und ihn dadurch in seiner eigentlichen graphischen Konfiguration verschieben; Bilder, Figuren, die in der Form des Schriftdokuments selbst neue Sinnbildungen in Gang bringen, die durch Variationen und Transformationen dieser Form neuartige, ungeahnte Sinn-Effekte oder Sinn als Effekt hervorrufen.

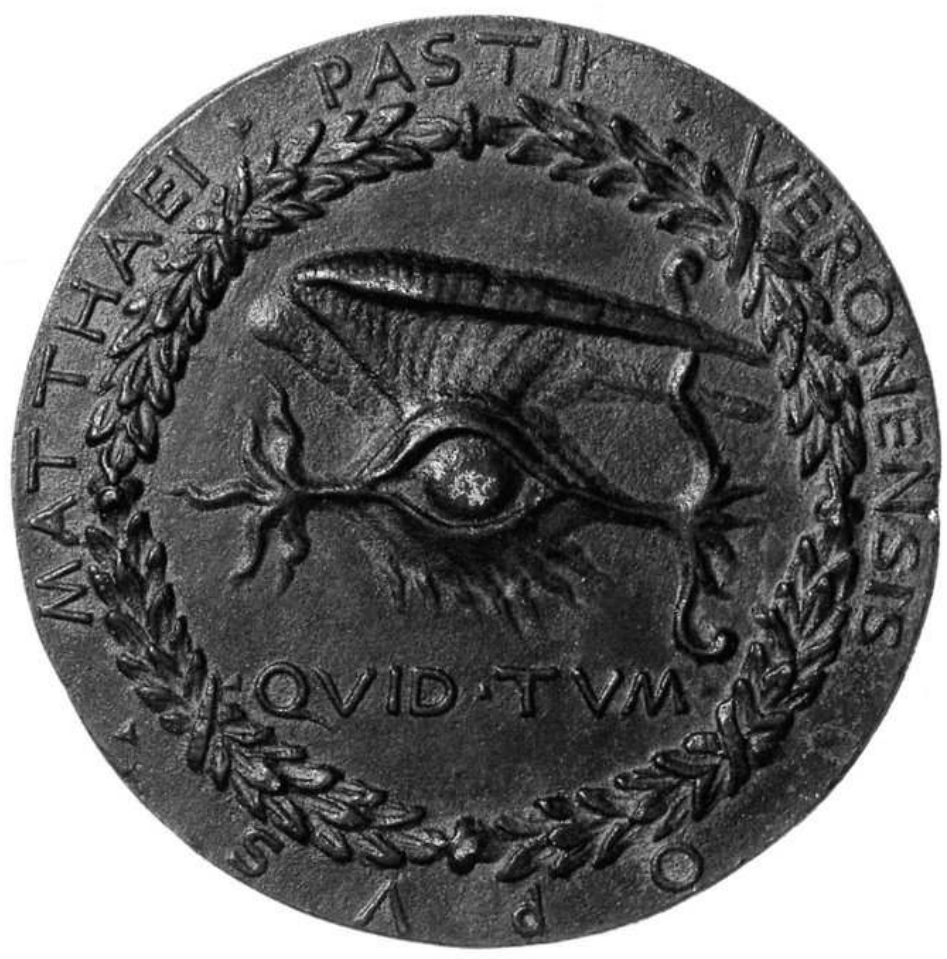

Matteo di Pasti, Rückseite der Alberti-Medaille: Quid Tum 


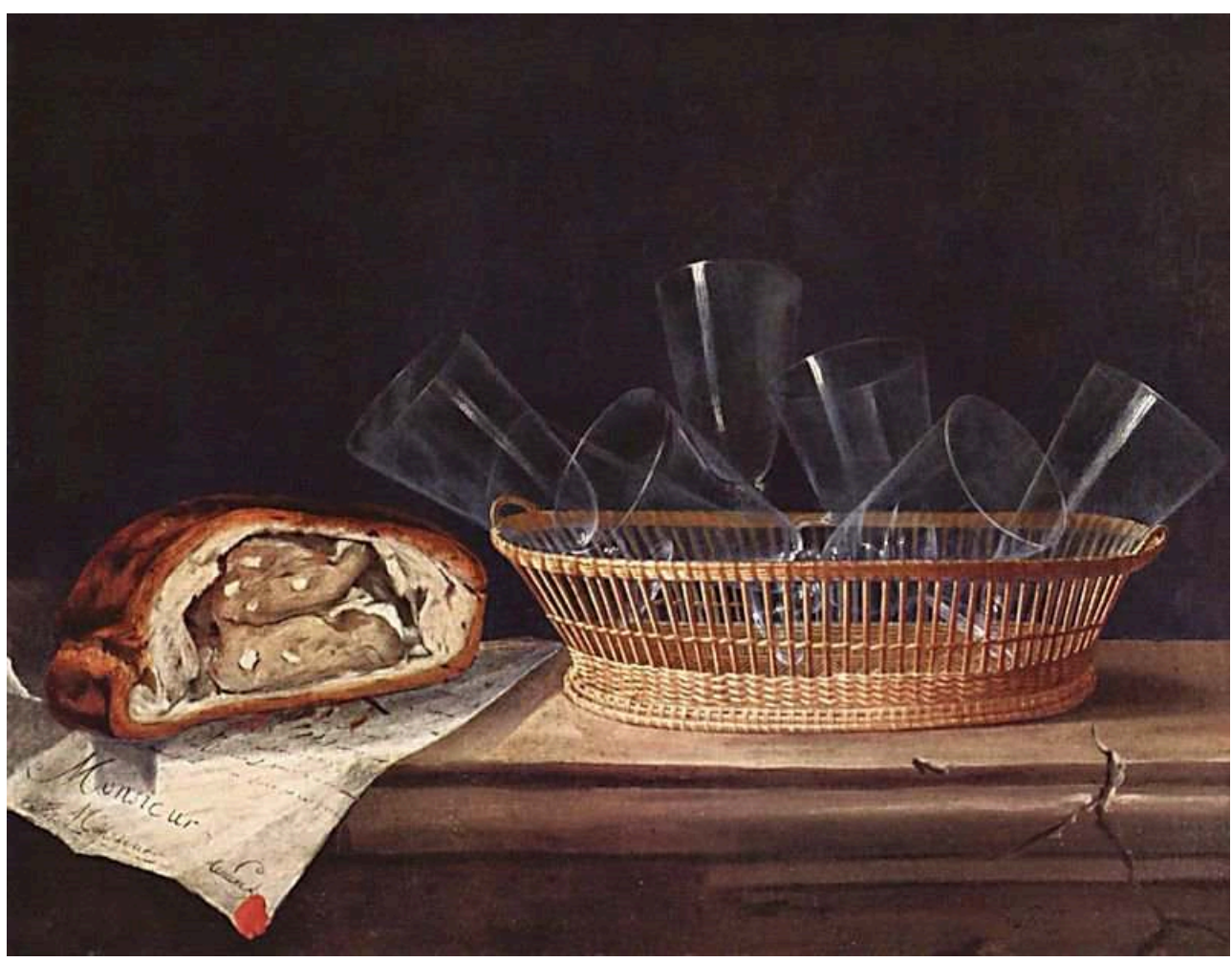

Sebastian Stoskopff, Corbeille de verres et pâté, 1644. Öl auf Leinwand, 49,5 x 63,5. Straßburg, Musée de l'Oeuvre Notre-Dame

Photo: Musées de la ville de Strasbourg, Mathieu Bertola

6 Kurz, die noch junge Semiologie der 60er Jahre hegte im Bereich der Bildsemiotik eine Vorliebe für solche hybriden Objekte, in denen eine sprachliche Nachricht und ein ikonisches Gebilde nicht bloß nebeneinandergestellt sind, sondern "symbiotisch" zusammenwirken in außergewöhnlichen konkreten Gefügen, innerhalb deren das Ikonische das Sprachliche in der Form und der Substanz seines Ausdrucks und seines Inhalts besetzt, oder umgekehrt das Sprachliche das Ikonische, auf ebenjenen vier Ebenen. So findet sich z. B. in der von Matteo di Pasti gravierten Alberti-Medaille eine unbestimmbare komplexe und bedeutsame Interaktion zwischen der Inschrift (nichts weiter als einer phatischen Floskel Quid Tum[Was nun]) und der Gestalt eines höchst beunruhigenden geflügelten Auges, dem merkwürdige Flammen entwachsen; oder wie die Vanitas oder das Stilleben von Stoskopff, wo man auf dem Umschlag eines Briefes den Namen des mit dem Maler befreundeten Antwerpener Händlers lesen kann, für den das Gemälde bestimmt war, und das den heutigen Betrachter an ein kleines Bild von Klee erinnert, welches einen an Kandinsky adressierten Briefumschlag abbildet, der darauf verweisen die durchgestrichenen Städtenamen - ihm durch ganz Europa folgt. Klee spielt hier zwischen »Text und Bild« etwas Einschneidendes, worauf Jasper Johns in The Flag zurückkommen wird. 

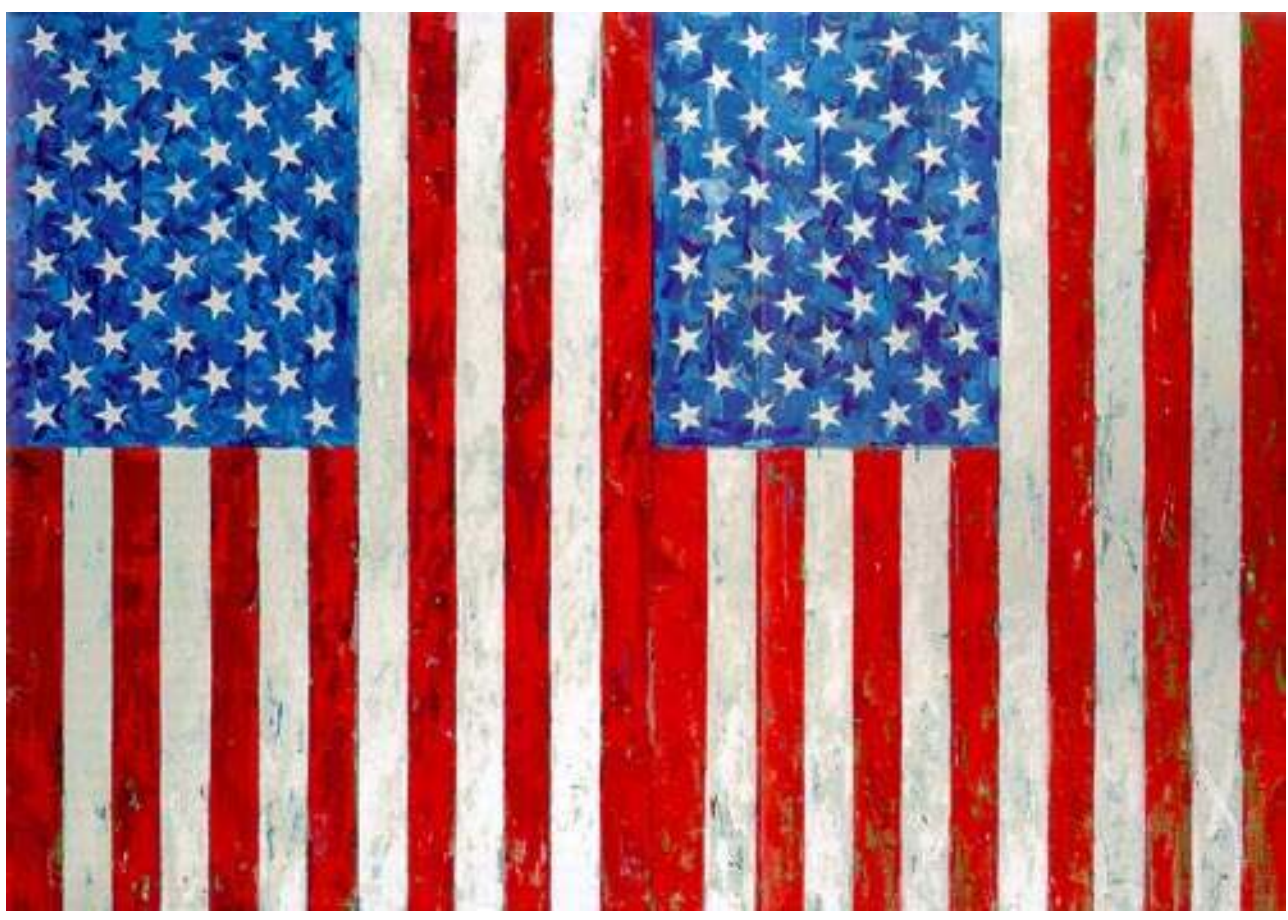

Jasper Johns, Two Flags, 1973. Öl und Enkaustik auf Leinwand, 145,9 x 176,7. Privatsammlung (C) Jasper Johns / ADAGP, Paris 2012

7 Dass derlei Fragestellungen nach wie vor aktuell sind, mag die Existenz einer englischen Zeitschrift beweisen, die diese Problematik bereits in ihrem Namen als genuine Disziplin der Kunstgeschichte und Kunsttheorie ${ }^{7}$ institutionalisiert, oder auch die neueste Ausgabe einer exzellenten Pariser Zeitschrift für Gegenwartskunst mit dem Titel »'art et les mots ॥ $^{8}$, die sich auf die Theorien einiger Arbeiten aus den sechziger Jahren stützt, dabei allerdings vergisst, sie zu benennen. Mag sein, es wurde im Eifer und wegen der relativen Unbefangenheit der Auseinandersetzung mit dem Thema schlicht vergessen.

Diese symbiotischen Objekte aus Schrift und Figur bieten, offen gesagt, den großen Vorteil, dass sie die semiotische Forschung im Bereich der visuellen Künste vor der Naivität, wenn nicht gar Arroganz oder Anmaßung bewahren, Grundlagen und Regeln des Lesens ohne Weiteres auf gemalte Bilder oder Erzeugnisse visueller Kunst $\mathrm{zu}$ übertragen. Allein die Existenz solcher Erzeugnisse verbietet es, in ihnen Konstituenten $\mathrm{zu}$ sehen, die für den Semiotiker aus den elementaren Universalien der Signifikation zu deduzieren wären. Mehr noch, diese merkwürdigen und vertrauten Objekte aus »Text und Bild« konstituieren in sich selbst ein kritisches Experiment einer solchen apriorischen Übertragung und Deduktion, desgleichen sie auch ein kritisches Experiment bezüglich der Begriffe: plastisches Zeichen, Bildvokabular, figurative Syntax, Grammatik der Stile einführen. Ich meine, es lassen sich mit ihnen die metaphorischen Werte dieser Begriffe einkreisen und ihre zulässigen Anwendungsbedingungen exakter bemessen: wie gesagt, metaphorische Werte, Werte der Verschiebung; zulässige Anwendungsbedingungen: zunächst ein kritisches Bewusstsein von dieser Verschiebung haben. Unsere symbiotischen Objekte aus TextBild / Schrift-Figur machen eine Arbeit sichtbar, eine wechsel- und gegenseitige Bearbeitung des Sprachlichen und des Ikonischen, wobei wir uns hier eine physische Arbeit vorzustellen haben: das Erzeugnis einer Kraft durch Verschiebung. Das kritische 
Experiment, welches Schrift-Figuren, Text-Bilder, das Lesbar-Sichtbare durch ihre spezifische Kreativität erlauben, bestünde darin, jene Verschiebungen des Sprachlichen und des Bildlichen möglichst exakt zu bestimmen, um - im Sinne von Nietzsche und Deleuze - »die Kräfte abzuschätzen«, die sie in Buchstaben, Wörtern, Sätzen, in Strichen, Linienführungen, Figuren, Flecken, Schraffuren und Konfigurationen bewirken.

9 So verweist das kritische Experiment, zu dem diese symbiotischen Objekte die semiotische Forschung einladen, zurück auf das schöpferische Experiment, bei dem diese Objekte zugleich Proben, Garanten und Erzeugnisse sind. Eine der Zielsetzung dieser Forschung könnte darin liegen, zu einer theoretischen und konzeptuellen Formulierung dieser Kritik des schöpferischen Experiments zu finden, vorausgesetzt jedoch, Kritik wird hier im höchsten, Kantischen Sinne verstanden: Die Werke der Schrift-Figur offenbaren dem, der sie wirklich sehen und lesen will, die Voraussetzungen des auf das Kunstwerk bezogenen Gebrauchs von Sprache und Diskurs wie auch die Grenzen dieses Gebrauchs. Kritisches Experiment, schöpferisches Experiment, semiotische und semantische Arbeit. Ich wollte in Bezug auf die Ausstellung "Picasso poète«, seiner kürzlich in Paris erschienenen Schriften, all diese Begriffe zugleich evozieren und habe deswegen die Wendung Laboratorium der SchriftFigur [laboratoire d'écriture-figure] gewagt.

10 Sehen wir uns diese Prämissen der Theorie und Methode noch einen Moment genauer an, anhand zweier Werke - das eine der Schrift angehörend, das andere der Malerei -, in denen aber dies schöpferisch kritische Experiment, die wechselseitige und gegensätzliche Arbeit von Schrift und Figur, in all ihren Kräften der Verschiebung offenbar wird. Das erste Beispiel ist ein Schriftgestus von Picasso aus dem Jahr 1935. Das zweite, sechzehn Jahre zuvor entstandene, ein Gemälde von Paul Klee.

11 Am 20. November 1935, nachdem er dieses Datum vermerkt hat, schreibt Picasso:

(1) 


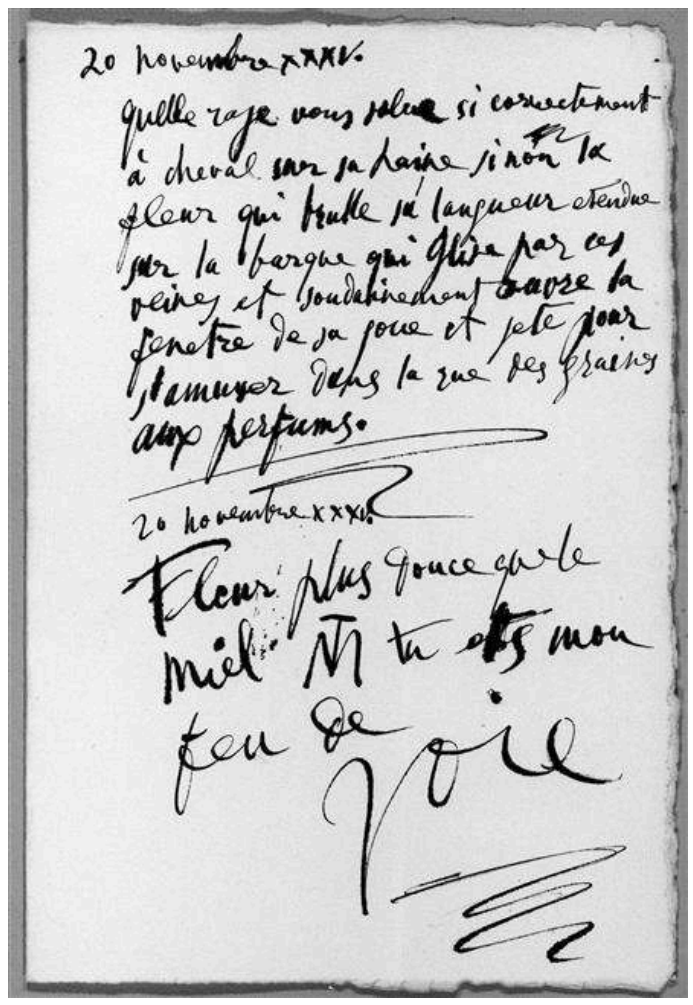

Pablo Picasso, Gedicht vom 20. November 1935, 3. Zustand

(c) Succession Picasso 2012, (c) RMN-GP 2012

Dieses Beispiels dient mir dazu, mich mit dem Sinn-Effekt zu beschäftigen, der sich

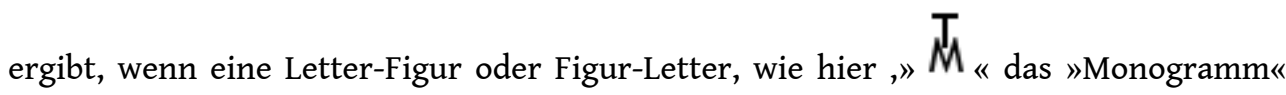
aus der Kombination der Initialen eines Namens, in einen geschriebenen Text eindringt. Die Letter-Figur, das Monogramm, verbirgt den Namen, besiegelt ihn in ihrer Kombination, gibt dabei aber doch die Produktionsmatrix an: die auf höchst individuelle Weise ineinander angeordneten Buchstaben $» M \ll$ und »T«. »M.T.«, MarieThérèse Walter, Picassos damalige Geliebte.

Die Letter-Figur schiebt sich exakt in die Mitte des Satzes. Sechs Wörter gehen ihr voraus, sechs folgen ihr. Gleichwohl besetzt sie nicht das visuelle und graphische Zentrum. Für den Blick dominiert das letzte Wort »Joie« den gesamten Text mit dem Stellenwert einer Signatur, nochmals bekräftigt durch den arabesken Federstrich, der sie mit einem energischen Schnörkel unterstreicht.

Erste Anmerkung: Eine genaue Transkription lässt die merkwürdige Zeilenaufteilung erkennen, die so gewollt sein muss, hätte doch das Papier dem Schreiber reichlich Platz gelassen, um den Satz entsprechend dem "Sinn" und den Gliederungen der Syntax niederzuschreiben, beispielsweise:

(2)

Fleur plus douce que le miel [Blume, süßer als Honig

(M) tu es mon feu de joie. (M) Du bist mein Freudenfeuer.]

Wir stoßen hier also auf eine zweifache Aufteilung oder zweifache Gliederung. Jene, bei der Lektüre "erwarteten", siehe (2), wird von einer anderen überlagert, siehe (1), die mit ihr spielt, sie im Schriftraum bearbeitet. Diese zweite Leseweise setzt "Blume«, »Honig" und «Feuer" an den Anfang der drei graphischen Zeilen, deren letzte sehr kurz ist, da das Wort »Freude« den Platz einer Signatur einnimmt (»Ich, die Freude«). 
Thnte Anmerkung: Die Letter-Figur $» M \ll$ ist auch Toi et Moi, du und ich, vereint im Freudenfeuer, ein kurzes, an M.-T. W., Marie-Thérèse Walter, gerichtetes Liebesgedicht, »Du bist« [»tu es«] ... Dieses »T« finden wir nun unterschwellig in der Schreibung des » « von »fleur« wieder (eine Blume, die Du [Toi Thérèse] schon bist), bevor es sich in das »M« von Marie pflanzt. Und wir finden es nochmals im Wort »ets«: es ist das »T« in dem was »Du bist« [»tu es«] und halbiert das Sein des »Du bist« [»tu es«]. So ist das Monogramm als Letter-Figur oder Buchstabe des Figuralen (mit Kant gesprochen) die optimale Annäherung an das Schema der reinen Einbildungskraft und zugleich der Buchstabe des Unbewussten in der graphischen Formation, realisiert in dem kurzen Satz eines Wortgedichts, der Liebe »macht«, sie buchstäblich vollzieht. Daher überrascht die »hyperbolische« Inversion, denn hier ist die Blume, aus der Honig gemacht wird, süßer als der Honig, der von ihr kommt. Die den Honig und die Blume verbindende semantische Affinität der Süße steht indessen im Gegensatz zum Feuer, das brennt, und dessen Verbrennungen man mit Honig behandelt. "Hinweg über« den Honig stehen Blume und Feuer sich in semantischer Opposition gegenüber; doch graphisch sind sich die beiden Wörter sehr nah - es fehlt nur der Buchstabe »l«, und die Blume wird Feuer oder das Feuer eine Blume.

das $» W \ll$ aus dem Namen Walter, dem Familiennamen von Marie-Thérèse. Die FigurLetter entwickelt sich durch Zusammenfügen und Umdrehen zu Marie-Thérèse Walter. Das »T«(hérèse) steckt in dem »M«(arie), und das »W«(alter) ist, zu einem »M « gedreht, ein zwischen den Beinen des »M« steckendes »T«. So sammelt die Biene den Blütenstaub der Blume, um daraus Honig zu machen. Der Buchstabe ist der Ausbruch von Picassos Liebesfeuer in der Blume von Marie-Thérèse, süßer als Honig.

Vierte Anmerkung: Das "T« ist aber auch der Degen des Toreros zwischen den beiden Hörnern des Stiers oder das Kreuz auf Golgatha zwischen den beiden Dieben, die Kreuzigung, die Picasso mit seinem Spott nicht verschont, indem er "Maria(Magdalena)« und den Gekreuzigten auf dem »T« (Kreuz) in einer blasphemischen Profanierung darstellt.

Trivium, $10 \mid 2012$ 


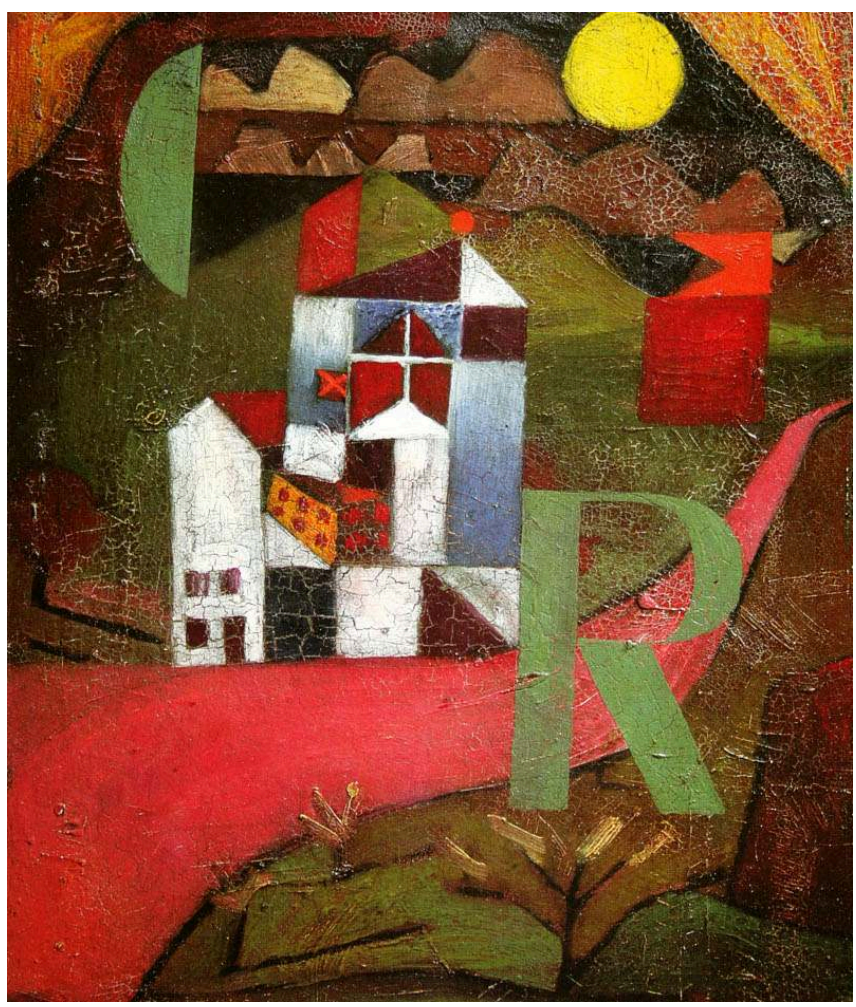

Paul Klee, Villa R, 1919. Öl auf Karton, 26,5 x 22. Öffentliche Kunstsammlung Basel, Kunstmuseum

Das zweite Beispiel ist Klees kleines Gemälde Die Villa $R$ (1919). Hier verläuft die Bewegung umgekehrt, denn es handelt sich nicht um ein geschriebenes Gedicht oder Lied, sondern um ein Gemälde - um ein ikonisches Ensemble zunächst, das von einem Buchstaben, dem Konsonanten »R« besetzt wird,der sich nur mit einem Vokal artikulieren lässt. Nun haben wir hier keinen Vokal - der Buchstabe ist stumm, sofern nicht das gesamte Gemälde ihn zum Sprechen oder Singen bringt. Ein Landschaftsbild mit einem sich nach hinten verlierenden Fluss oder Weg. Am Rand des Weges oder Flusses eine Villa. Das auf das Landschaftsbild gesetzte rote "R« hebt die Tiefenwirkungen auf, insbesondere jene, die der Fluss oder der Weg erzeugt. Der Buchstabe lässt das Gemälde als Schauspiel [spectacle] erscheinen; er steht auf der transparenten Aufführungsfläche, die auch das Stück Vorhang oben rechts kenntlich macht; das ganze Gemälde wird ideogrammatisch: Durch den Buchstaben tendiert es zur Schrift, zur Fläche, eine Trübung der Transparenz, als wäre es auf die Fläche gemalt. Gleichzeitig gibt es aber auch eine gegenläufige Strebung: Tatsächlich entbindet das Gemälde den Buchstaben von seiner Buchstäblichkeit und wandelt ihn in eine »schreitende« Figur - das »R« verlässt auf seinen Beinen den Weg oder den Fluss, um querfeldein zu gehen.

Das Klee-Beispiel - dem sich noch viele hinzufügen ließen - zeigt uns, wie das Eindringen eines Buchstabens in ein ikonisches Ensemble ein kritisches Experiment für dieses Ensemble schafft, insoweit es die Bedingungen der Repräsentation selbst ans Licht bringt: Der Buchstabe ist - um in der Sprache des Labors zu reden - ein Operator, ein Katalysator für die Opazität oder die Trübung der mimetischen Darstellung. So wie umgekehrt im selben Beispiel die Attraktion der figurativen Darstellung des Buchstabens diesen ins Mimetische oder Darstellende driften lässt. Die Letter, der Druckbuchstabe - eine römische Majuskel - ist nicht mehr die konventionelle 
Transkription eines Phonems, sie wird durchscheinend in mimetische Darstellung: Der Buchstabe $» R \ll$ stellt eine schreitende Figur dar.

Nun ist es gewiss interessant, anhand dieser historisch unterschiedlichen Beispiele, die jedoch beide mit Schrift und Figur experimentieren, die komplementären Schlüsselbegriffe Opazität und Transparenz des Zeichens und der Repräsentation genauer darzulegen, die wir in ihren philosophischen Grundzügen in der Neuzeit deutlich vorgezeichnet finden. Für die Philosophen des klassischen Rationalismus ist der Gedanke als Ganzes ein Zeichen. So heißt es bei den Logikern von Port-Royal:

"Man nennt Vorstellen die einfache Ansicht der Dinge, die sich unserem Geist darbieten, wie wenn wir uns eine Sonne, eine Erde, einen Baum, einen Kreis, ein Viereck, das Denken, das Sein vergegenwärtigen, ohne uns von ihnen eigens ein Urteil zu bilden; und die Form, unter der wir uns diese Dinge vergegenwärtigen, heißt Idee. " ${ }^{9}$

Die Idee ist ein Zeichen, das heißt, ein Ding, das etwas anderes darstellt. Vermittels der Zeichen, welche die Ideen sind, erkennen wir die äußere Welt. Doch die Funktionsweise der Zeichen weist ein Paradox auf. Nehmen wir zum Beispiel das Lesen: Wenn ich lese, bin ich mir der Buchstaben nicht bewusst, mein Bewusstsein nimmt allein die Ideen wahr, deren Zeichen die aus Buchstaben gebildeten Wörter sind, und trotzdem eröffnen sich mir die Ideen nur vermittels der Zeichen. Richte ich allerdings, umgekehrt, meine Aufmerksamkeit auf die Buchstaben selbst, weiß ich sehr schnell nicht mehr, was sie repräsentieren. Das operierende Zeichen ist zugleich abwesend und anwesend, transparent und opak. Wenn es bei den Logikern von Port-Royal heißt: »die heiße Asche verbirgt, als Ding verstanden, das Feuer und als Zeichen offenbart sie es « ${ }^{10}$, so zeigt dies das doppelte Charakteristikum der Repräsentation: ihre Transparenz und ihre Opazität.

Das theoretische Experimentieren mit Texten und "Bildern«, Zeichen und Repräsentation, Schrift und Figur, als Betätigungsfeld der zweiten Generation dieser Semiotik, würde die aus der Opazität des Zeichens und der Repräsentation hervorgehenden Probleme genau in Betracht ziehen, insbesondere die seiner Materialität in den verschiedenen Ausdruckssubstanzen und entsprechend ihrer Organisation (Stimme, Schriftzug, Zeichnung, Farbe etc.) sowie die Wirkungen dieser Materialität auf die Phantasie, das Empfinden, die Lust von Auge und Ohr. "Der Ton der Stimme macht auch auf den Weisesten Eindruck«, notiert Pascal in Anlehnung an Montaigne. Oder um Brunelleschi und Alberti, die beiden Kunsttheoretiker der Renaissance, zu bemühen: Das Gemälde ist ein offenes Fenster zur Welt; es stellt sie kraft seiner Transparenz tatsächlich dar. Damit dies aber gelingt, muss es sich als Schirm [écran] zwischen Betrachter und Welt schieben, als Träger und Konstruktionsoberfläche für den illusorisch tiefen Raum, als Fläche, worauf die Figuren ihre Geschichte entfalten werden. Und die Künstler werden Träger, Oberfläche und Fläche oder ganz allgemein all jene nicht-mimetischen Elemente der mimetischen Darstellung einsetzen, um ihnen politische, soziale oder theologische Bedeutungen einzuschreiben, welche die Darstellung nicht einbeziehen konnte. Jedes Zeichen, jede Darstellung bezeichnet sich, bedeutet sich, bezieht sich - eben darin liegt die Definition seiner Opazität: Das Zeichen stellt sich selbst und im gleichen Zuge etwas anderes dar, und in dieser reflexiven Bezogenheit verweist jedes Zeichen, jede Darstellung auf ein praktisches Ausdrucksvermögen oder, um es mit Kant zu sagen, auf ein "Ich denke«, das alle Vorstellungen [représentations] begleitet und das heute in ein "Es denkt» umzuformulieren wäre, eine »Intention«, die in jeder sprachlichen Äußerung und jeder 
bildlichen Darstellung zum Ausdruck kommt, was diese Zeichen und Figuren allein durch ihre Existenz bezeugen.

Ähnlich zur Bearbeitung der abbildenden Transparenz des Bildes durch seine zeigende oder reflexive Opazität könnte es ebenfalls die Bearbeitung der darstellenden Transparenz des Diskurses, Textes, Satzes, Wortes, Zeichens geben, die indes noch transparenter sind, da ihrem ganzen Wesen nach konventionell und institutionell: »unmittelbare« Transparenz, Bearbeitung durch die opaken Grenzen des Diskurses. Rhetoriker und Poetiker, von Gorgias bis Fontanier, lernten nicht zuletzt bei den Rednern und Dichtern und deren wirksamsten Schöpfungen, den Redefiguren der Hypotypose, der Harmonik, des Subjecto ad aspectum, der Alliteration, welche, so schreibt einer von ihnen, die Dinge so lebendig, kraftvoll und beseelt schildern, dass man beim Hören der Wörter glaubt, sie zu sehen. Der jedoch darüber hinaus nicht bemerkt, dass, wenn Perioden und Strophen, Sätze und Verse, Wörter, Konsonanten und Vokale vor Augen führen, wenn also Sprache sehen lässt, dies durch die Kraft geschieht, die sie durchdringt und die durch ihre hierarchisierten Organisationen artikuliert werden; durch die Kraft, welche sozusagen ihre eingeführte Transparenz [transparence instituée] verschiebt: Zeichen und Buchstaben, Wörter und Sätze gestalten mit dem Fleisch, dem Stoff der Stimme, dem Klang der Worte, wie es Poussin in Anlehnung an die italienischen Musiktheoretiker des 16. Jahrhunderts nannte, um daraus die Analogie $\mathrm{zu}$ den eigentlichen pikturalen Modi der Farben und der Anordnung der Figuren herzustellen. Diesseits und jenseits der Wörter und Sätze zeichnet die Kraft dieser sprachlichen Figuren in den Körper des Werkes - sei es ein malerisches oder ein sprachliches - die opake Syntax des Begehrens ein, das den Maler oder Redner erfüllt, sowie dessen pathetische Wirkungen, die ihrerseits im Körper des Betrachters und des Zuhörers ihren Ort haben. Für das theoretische Experimentieren der Semiotik gilt es also, die Wirkungen dieser Kräfte der Opazität, der Präsentation der Repräsentation im Auge zu haben, die Wirkungen, in denen die imaginären Identifikationen des Subjekts Gestalt annehmen.

Es muss nicht erwähnt werden, dass unsere symbiotischen »Text-Bild«-Objekte konkrete "schöpferische" Experimente darstellen, in denen sich verschiedene Arten und Formen von Beziehungen zwischen Substanzen und Formen des Inhalts und Ausdrucks unterschiedlicher Gattungen finden: Sonach wird mit ihnen die Werkstatt »Picasso als Dichter« zu einem Versuchslaboratorium für die Paarung von Heterogenen und der Erzeugung ihrer Effekte. Man muss an dieser Stelle zweifellos einräumen allerdings in der Absicht, es zu problematisieren und zu theoretisieren -, dass zwischen dem Experimentieren im Laboratorium der Semiotik oder vielmehr dem der Theorie und dem des Künstlers im Laboratorium der Schrift und der Figur ein fundamentaler Unterschied besteht. Ich komme späterhin darauf zurück. Im Laboratorium des Künstlers »denkt es« [»ça pense«]. Die Kunst denkt im Ausdruckswillen und in der Ausdrucksabsicht des Dichter-Malers, doch wird sich dieses Denken in all seinen Gestaltungen und Ausführungen immer mit und in einem Sinn konstituieren - Sinn, verstanden gemäß den Paradigmen erstens der eigentlichen semiotischen Bedeutung, zweitens des ästhetischen Empfindens und drittens des pathetischen Gefühls: ein Sinn, der im Werk, im Erzeugnis, vom gemalten Bild bis zum geschriebenen Gedicht, seine Verkörperung findet, in der wahrsten und allumfassenden, um nicht zu sagen theologischen Bedeutung des Wortes. 
Untersuchen wir als Beispiel eines solchen »schöpferischen« Experiments aus dem Laboratorium der Schrift-Figur eine jener komplexen Arbeiten Picassos. Augenscheinlich kommt in dem gewählten Beispiel ausschließlich Schrift zum Einsatz, doch mit dieser Schrift wird "gespielt", das Werk bearbeitet sie - verschiebt sie durch die begehrende Kraft - in der Wiederholung, verstanden als Variation desselben und Transformation des Unterschieds. Ich möchte über die Serie der elf Schriften Il neige au soleil [es schneit in der Sonne] sprechen, unten links datiert mit »Paris 10 janvier XXXIV« und nummeriert I bis XI. Was mag das Analyseprotokoll, ich meine das Protokoll des theoretischen semiotischen Versuchs, über diese Schrift-Arbeit (oder dieses SchriftSpiel) ergeben? Christine Piot, eine der Organisatorinnen der eingangs erwähnten Ausstellung, sagt ganz richtig:

»Der Satz vom 10. Januar 1934 zeigt, dass sich (zeitliche) lineare Schrift zu einer (räumlichen) Zeichnung entwickeln kann.«

Als weitere Beispiele können noch Zeichnungen aus der Serie Royan (1939-1940) sowie die im Februar 1935 entstandenen Porträts von Jaime Sabartès angeführt werden. Es empfiehlt sich, die Recherche als eine Untersuchung des Variationsprozesses eines identischen Syntagmas anzulegen, das einen vollständigen grammatischen Sinn ergibt: »l neige au soleil.«

Dieser allmählich sich vollziehende Variationsprozess der Schrift beruht, im Sinne ihrer Skription, auf einem Dispositiv der Transformation von Schrift in eine Zeichnung, der Transformation von dieser schriftlichen Textsequenz in eine Figur oder vielmehr in eine visuelle graphische Konfiguration. Die Untersuchung des Variationsprozesses beschäftigt sich hier mit einem derselben Substanz und derselben Ausdrucks- und Inhaltsform zugehörigen semiotischen Ensemble, dem der Sprache.

Die Sprache hingegen wird, und das ist paradox, zugleich Erzeuger und Erzeugnis eines Transformationsdispositivs, das seinerseits zwei heterogene semiotische Ensembles (das Text-Bild; die Schrift-Figur; das Sprachlich-Ikonische) unterschiedlicher Substanzen und Formen des Ausdrucks und des Inhalts betrifft. Unser theoretisches Experimentieren im Laboratorium Picasso sollte nun aber nicht allein darauf abzielen, die sukzessiven Operationen von Prozess und Dispositiv in den elf Proben oder Stationen des Experiments $\mathrm{zu}$ beschreiben. Es sollten auch die Regeln des Variationsprozesses sowie die Prinzipien des Transformationsdispositivs extrahiert werden oder, kurz gesagt, die Struktur des Werkes Il neige au soleil, jener 11 Bögen Arches-Papier (26 x 32,5), auf denen sich mit Tusche geschrieben der Satz (die Sätze) »il neige au soleil« findet (finden). Doch ist ebenso klar - und hier möchte ich noch einmal den Unterschied zwischen dem Laboratorium des Theoretikers und dem der Künstlers unterstreichen -, dass das theoretische, semiotische, analytische Experimentieren den alleinigen Sinn und Zweck hat, den Gedanken des Werkes auf den Begriff zu bringen oder auch die Dynamik der Wiederholung (der Gleichheit und des Unterschieds), die Dynamik der Schrift und der Graphie, der Schöpferin dieses Werks, als Variationsregeln und Transformationsgrundlagen $\mathrm{zu}$ formulieren. Womit immense theoretische und praktische Probleme einhergehen. Ich werde mich damit begnügen, einige Forschungshypothesen festzuhalten. 


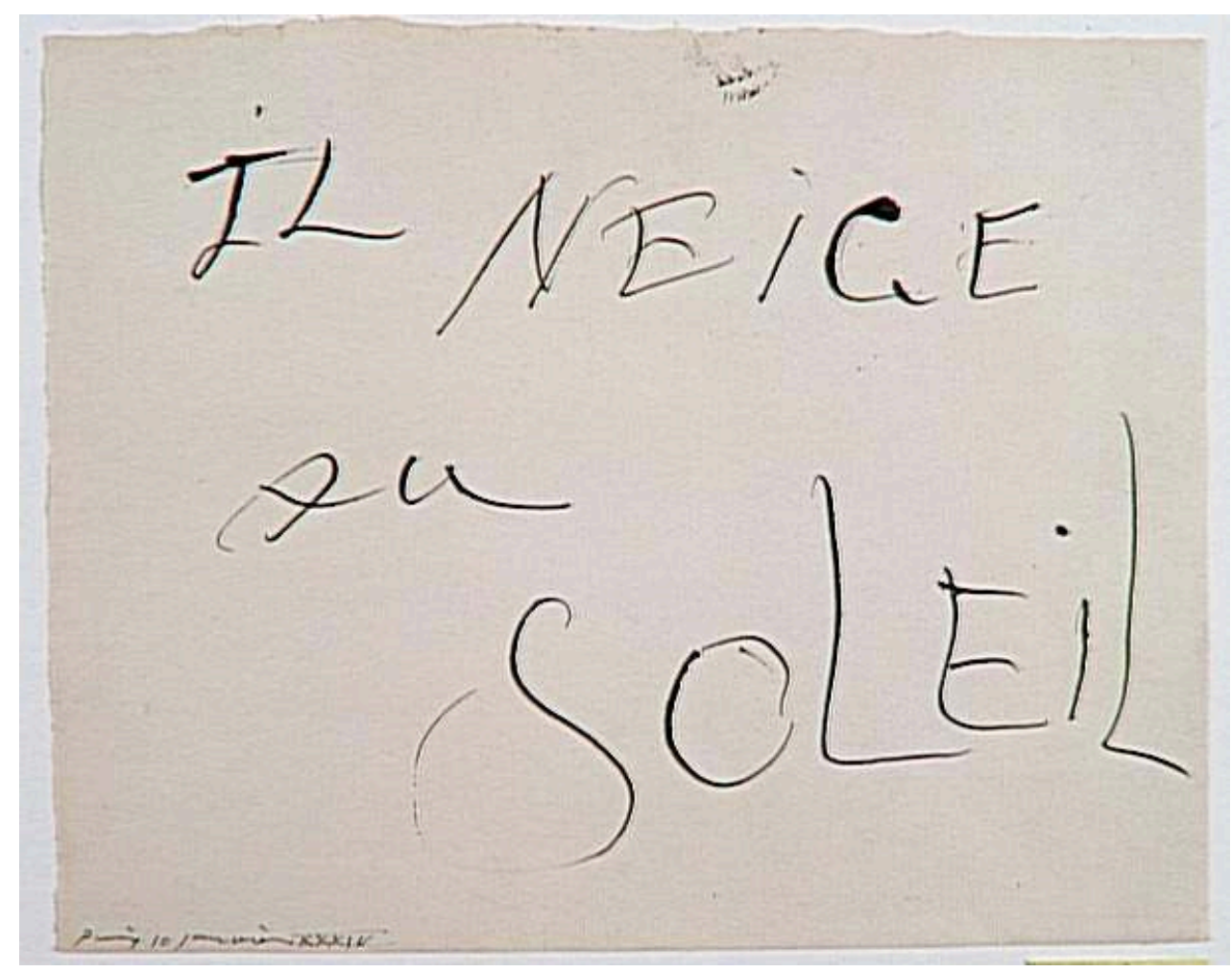

Pablo Picasso, I/ neige au soleil, 1934. Blatt 1, Tusche auf Arches-Papier, 26 × 32,5. Paris, Musée Picasso

(c) Succession Picasso 2012, (c) RMN-GP 2012

Erste Anmerkung: Zwar haben wir auf allen »Proben« [»épreuves«] die »lineare« Schrift des Satzes, doch läuft diese Schrift in der ersten Probe über drei Zeilen: il neige / au / soleil /; in der zweiten über zwei: il neige / au soleil; in der dritten über vier: Il / neige / au / soleil; in der vierten wiederum über drei, allerdings in anderer Weise als in der ersten Probe: il / neige au / soleil ... Wie die Sprache ist auch der Satz eine quantifizierte Einheit, und im "poetischen « Satz tritt Ikonismus auf, wenn sich die Markierungen der Quantität in der Sprache und die Zeichnung verbinden und einen einzigen Effekt schaffen - anders gesagt, wenn sprachliche Mengen Linien, Oberflächen, Schnitte und Trennungen, Felder und Umrandungen werden, was durch die Mediatisierung der Schrift geschieht. Um nun auf unseren "Satz" zurückzukommen, wir haben hier zwar die lineare Schrift ein und desselben Satzes, doch setzt sich dieser aus vier Elementen zusammen, aus vier diskreten Quantitäten Wörtern -, und diese aus Buchstaben: zwei, fünf, zwei, sechs. Ihre Zeilengruppierung ordnet sich auf dem Trägermaterial an, indem sie Oberflächen, unterschiedliche Schnitte und verschiedene Einteilungen festlegt.

Zweite Anmerkung: Natürlich ist die Schrift linear, auch zeitlich linear, doch die Schrift und die Wiederholung der identischen Satzaussage lässt in der Verschiedenheit ihrer Schnitte, Trennungen, Leerräume, Stellungen, Korrelationen, Parallelismen, Zeilensprünge, ja sogar Permutationen oder Verlagerungen kleinerer Einheiten innerhalb des Schriftkörpers des Gesamtsatzes und seiner Subeinheiten, den Wörtern, die Schriftzeile in fortlaufende Variationen und räumliche Neuanordnungen zerfallen, und damit zerfällt auch die Zeit der Linearität des Satzes, sie zerbricht; hier findet sich nicht mehr die sukzessive lineare und homogene Zeit, bestehend aus einer Kette von Zeitpunkten oder von "Jetzt«, die auf einer geraden Linie aufgereiht sind - die 
abendländische metaphysische Vorstellung von Zeit. Es zerbricht diese Linearität zwei Mal, ein erstes Mal zunächst in jeder Probe, d. h. in jedem Experiment der schriftlich geäußerten Aussage, nicht nur gemäß den »Zeilen«, sondern gemäß den »Lagen« auf der Oberfläche sowie den »Trennungen« und den »Leerräumen«; und ein weiteres Mal im gesamten Werk, auf den elf Arches-Blättern, das heißt gemäß der Wiederholung der gleichen Aussage »il neige au soleil« in den Stellungen und Gegebenheiten (die Begriffe sind hier im wortwörtlichen, räumlichen Sinn $\mathrm{zu}$ verstehen) unterschiedlicher Äußerungsakte. Eine zersplitterte Epiphanie: die Zeit eines jähen, unvermittelten Zusammentreffens von elf Kairos, elf Gelegenheiten, deren jede alle Zeiten im Präsens der Probe zusammenführt und die in der Gesamtheit mit ihren Variationen und Transformationen eine trans-figurierte Zeit der Schrift konstruieren.

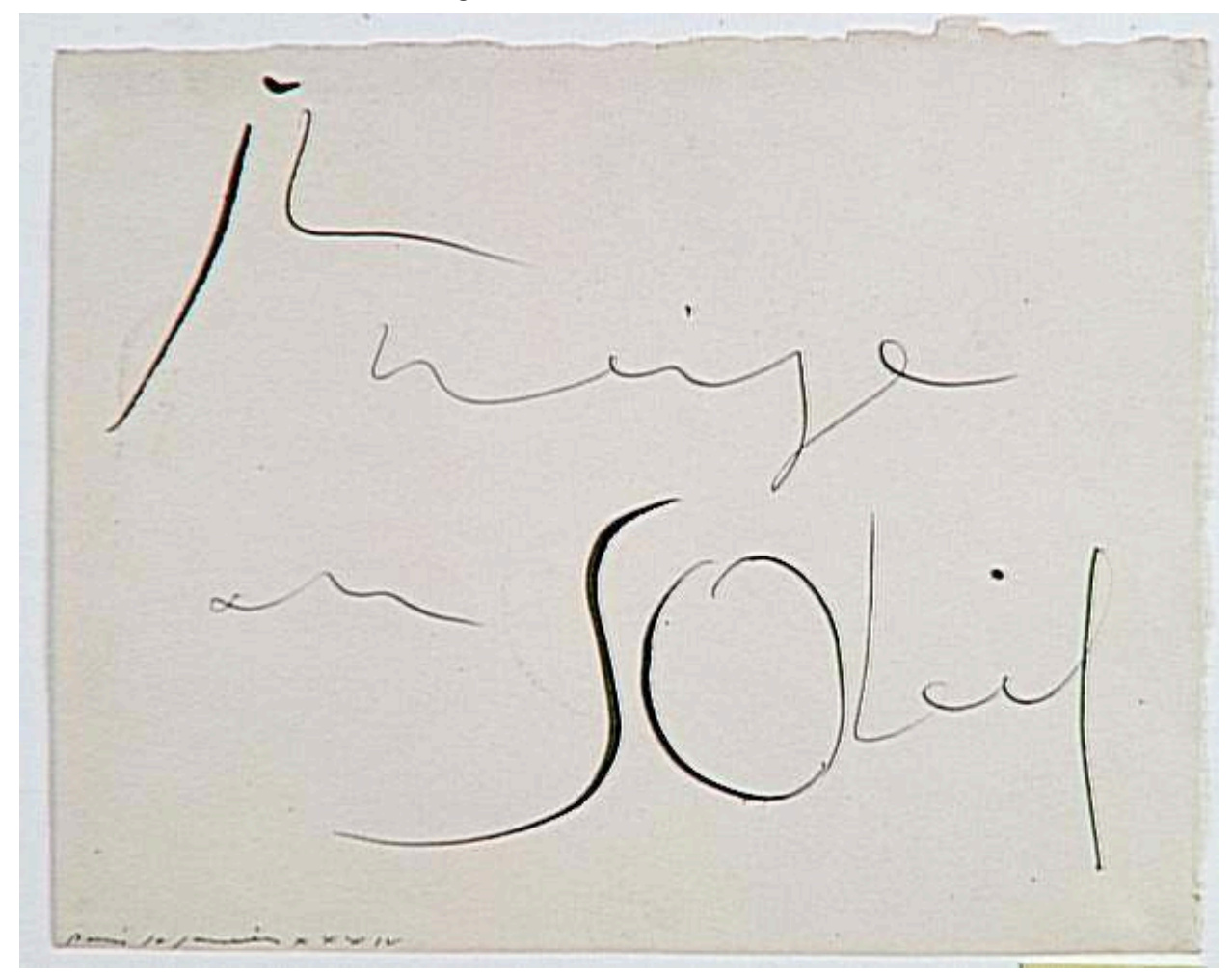

Pablo Picasso, I/ neige au soleil, 1934. Blatt 5, Tusche auf Arches-Papier, 26 × 32,5. Paris, Musée Picasso

(c) Succession Picasso 2012, (c) RMN-GP 2012

Dritte Anmerkung: »il neige au soleil« ist eine temporale Aussage über das momentane Wetter [temps] 1.) im grammatischen Sinn von tense: Indikativ Präsens; 2.) im semantischen Sinn von time: der gegenwärtige Augenblick einer Äußerung eines Ereignisses; und schließlich 3.) im kosmischen Sinn von weather, welches die meteorologische Präsenz der physikalisch merkwürdigen Konjunktion von Schnee und Sonne anzeigt. Die Paarung der Heterogene von Schrift und Zeichnung zielt darauf ab, diese physikalische Heterogenität, dieses kosmische Oxymoron oder, umgekehrt, die poetische Bildhaftigkeit oder die ein kosmischer Meteor gewordene barocke Rhetorik für die Lektüre einzufangen und für das Auge zu erzeugen. Doch wie können hier die Ausdrucksform in ihrer schriftlichen Transkription und die Inhaltsform in ihrer semantischen Artikulation wenn nicht zur Deckung kommen, so doch zumindest sich gegenseitig hervorbringen? Weiter gefragt, wie kommt es, dass die Variationen der schriftlichen Form, indem sie den Inhalt kontinuierlicher Sinneffekte erzeugen, uns 
nicht nur die Variationen des kosmischen Meteors (des Referenten), den der Satz »il neige au soleil« bezeichnet, sehen und begreifen lassen, sondern uns auch den Zugang zum wechselseitigen Dispositiv der Transformation von Schrift und Figur eröffnen?

Zumal, vierte Anmerkung, das meteorologische poetische Oxymoron "neige soleil« auf der Ebene des Graphs und der Stimme die Gegensätzlichkeit, welche die Figur definiert, wiederholt: Beide Wörter, ersteres ein Verb-Nomen, letzteres ein Nomen, beinhalten graphisch gesehen denselben zusammengesetzten Laut ei - bei neige am Anfang nach dem ersten Buchstaben $n$, bei soleil am Ende vor dem letzten Buchstaben $1-$, und auf manchen Proben weist der Laut sogar eine absolut identische Graphie auf. Geht man hingegen nicht mehr vom Visuellen aus, sondern vom Klanglichen, von der Stimme und vom Ohr, so stehen sich das e von neige (nez) und das ej von soleil (solej) als Vokal und Diphthong mit einem gemeinsamen Tonelement $e$ gegenüber. Wir haben also durch die beiden Kategoremata der Aussage »neige-soleil«, deren graphische Mengen bis auf einen Buchstaben identisch sind - fünf für »neige« und sechs für »soleil« -, deren phonische Mengen jedoch stark voneinander abweichen, sich nahezu verdoppeln: ne3/solej eine Wiederholung Desselben und der Verschiedenheit.

Fünfte Anmerkung: Verfolgen wir diese Betrachtung über stemps in seinen drei Bedeutungen tense, time, weather und die Funktionsweise des kosmischen Oxymorons auf ebenjenen Ebenen weiter, so werden uns die graphischen Variationen des ersten Wortes »il« auffallen, das sich im ersten Experiment noch nach dem Wort »neige« ausrichtet, einem Verb in der dritten Person Singular Präsens, dessen unpersönliches Subjekt es ist, »il neige", eine schriftliche semantische Gruppe (il neige), das sich jedoch in den nachfolgenden Experimenten von ihm isoliert: »il« - um durch seine schriftliche Loslösung auf die plötzliche Erscheinung des kosmischen Seins hinzudeuten, die danach nur vom physikalischen Meteor »neige au soleil« bestimmt wird und durch die Charakterisierungen, die diese Wörter in Position des Verbs und der Umstandsbestimmung auf der semantischen Ebene einführen. Beiläufig sei bemerkt, dass dieses theoretische Neu-Schreiben, mit dem ich Picassos eigenes Neu-Schreiben begleite, auf Benvenistes Analyse der Satzkerne rückverweist, welche im Wesentlichen die Analyse der klassischen Grammatiker aufnimmt: „Der Vogel fliegt« [»'oiseau vole«] wird in der Neu-Schreibung »il (est) l'oiseau volant« [»er (ist) der fliegende Vogel«], in der nun »oiseau volant " [»fliegende Vogel«] in einer Art Apposition zu einem unpersönlichen »er ist« [»il est«] steht, eine Epiphanie des Seins in der Sprache, welche die Charakterisierungen und empirischen Bestimmungen artikulieren. Schließlich möchte ich noch festhalten, dass in den letzten Proben das »il« gewissermaßen nach und nach zuerst vom »S« und dann vom »0« von »soleil« einverleibt wird. 


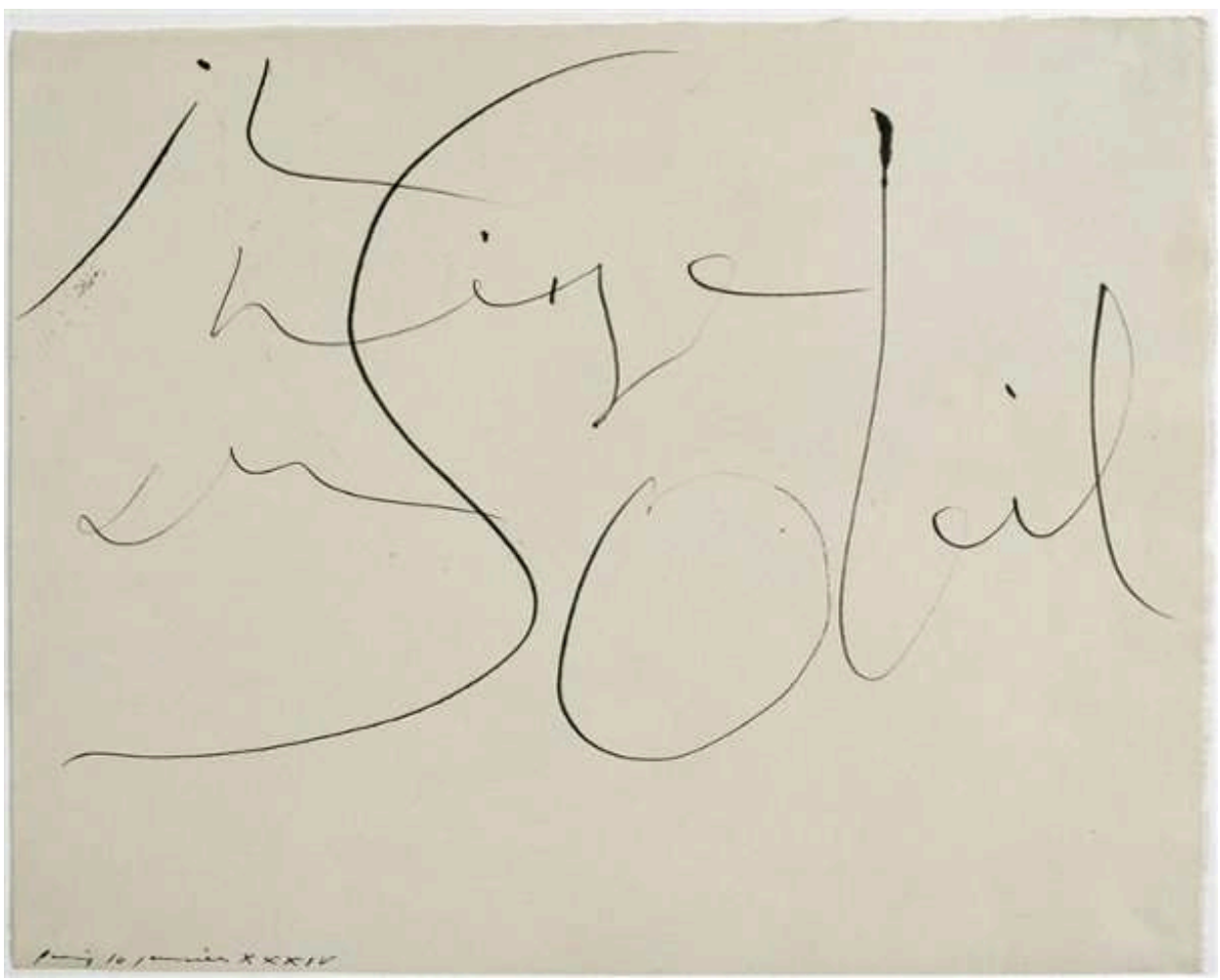

Pablo Picasso, I/ neige au soleil, 1934. Blatt 7, Tusche auf Arches-Papier, 26 × 32,5. Paris, Musée Picasso

(c) Succession Picasso 2012, (c) RMN-GP 2012

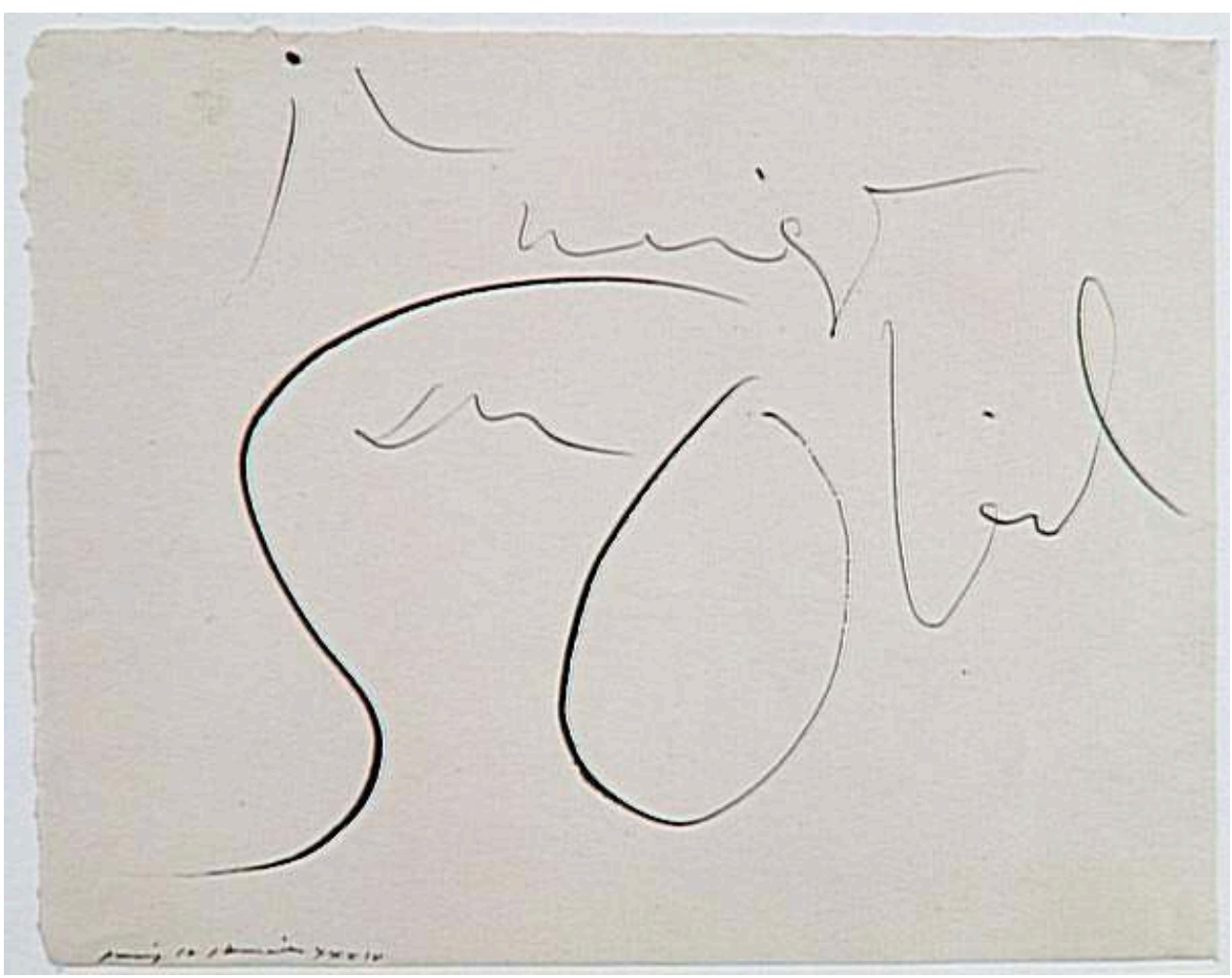

Pablo Picasso, I/ neige au soleil, 1934. Blatt 9, Tusche auf Arches-Papier, 26 × 32,5. Paris, Musée Picasso

(c) Succession Picasso 2012, (C) RMN-GP 2012 
Sechste Anmerkung: In Weiterführung der vorigen Anmerkung sollte die Analyse nun die graphischen Variationen der Buchstaben, aus denen sich die Wörter der Aussage »il neige au soleil« zusammensetzen, in Betracht ziehen, um zu untersuchen, welche Konnexionen und Beziehungen sie in den einzelnen Proben zwischen den verschiedenen Wörtern einleiten und welche Sinn-Effekte sie erzeugen, sieht man sich die vollständige Serie der elf Proben an. An eine erschöpfende Analyse darf hier allerdings nicht gedacht werden. Halten wir nur fest, dass die Buchstaben $\gg$ « und $» 0$ « aus dem Wort Soleil nach und nach immer kraftvoller und expressiver auf dem Trägermaterial hervortreten: bis in der 5. Probe das ॥ ««, gleich einer riesigen Akkolade, das Synkategorem "au«, den semantischen Konnektor, das graphische und syntaktische (syntagmatische) Bindeglied des Oxymorons "neige soleil« aufnehmen wird, um im Weiteren das Verb-Nomen neige zwischen $n$ und $e$ zu durchtrennen, sich an den unteren Teil des $i l$ zu hängen, das $a$ von au zu durchqueren und schließlich die gesamte Aussage mit einer großen, in einem Punkt das »0« von »Soleil« tangierenden Arabeske nach links zu versetzen. Betrachtet man das »0«, das einzige »0«, das der Satz enthält, so stellt man fest, dass es seine Position und seinen Platz als zweiter Buchstabe des Wortes Soleil aus der ersten Probe sehr schnell aufgibt, um eine fortschreitende Unabhängigkeit und Autonomie oder zumindest eine Evidenz, eine Prägnanz zu gewinnen, die darauf hinausläuft, dass es in der elften und letzten Probe "neige au« in seinen Kreis einschließt.

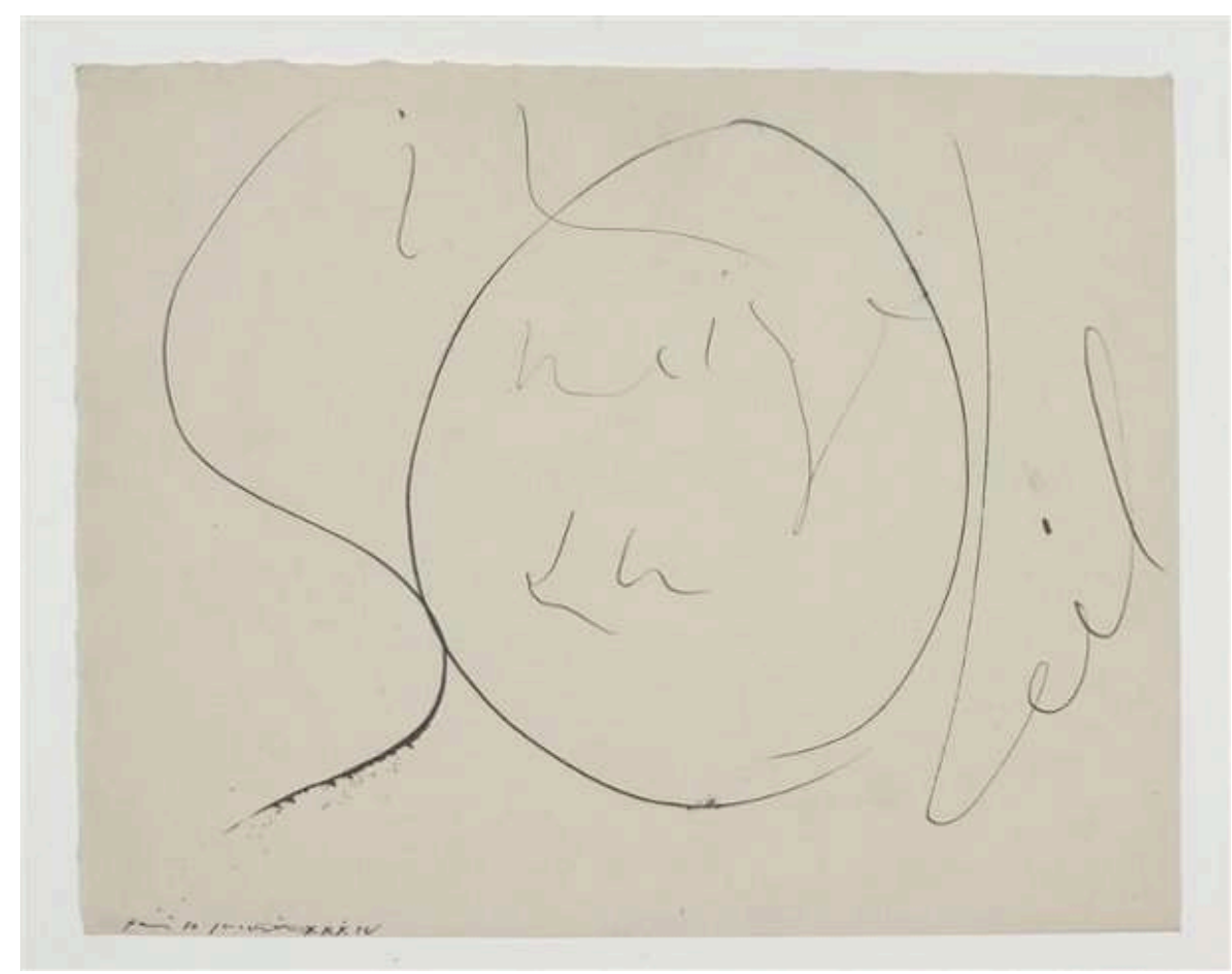

Pablo Picasso, I/ neige au soleil, 1934. Blatt 11, Tusche auf Arches-Papier, 26 × 32,5. Paris, Musée Picasso

(C) Succession Picasso 2012, (c) RMN-GP 2012

Zum Schluss kann man eine Beschreibung dieser letzten Neu-Schrift von »il neige au soleil« versuchen: Im Zentrum steht der Sonnenkreis des "0«, der sich "neige au« einverleibt und sich das »l« von »il« angehängt hat. Diesen Kreis begrenzt von links die peitschenförmige Akkolade des "S« und von rechts die grazile Linie des "L«, die 
ihrerseits in einem rankenhaft aufsteigenden Federstrich endet: »eil«, das nicht zum "0« gehörende "eil«, ein Parergon des Sonnen-»O«, ein äußeres graphisches - ironisches - Echo, aufleuchtend wie eine Vokalise, mit dem überdeutlichen Punkt des $i$, als Echo auf "ei« von «neige«, das vom "n" und "ge« verschluckt wird und dessen Traktus sich auflöst, um in der Klarheit, Deutlichkeit und Prägnanz des »0« zu verstummen. Ich verweise auf unsere vierte Anmerkung. Hinzu kommt eine graphische Variation, welche die Weisungen der Stimme und der Schrift bestätigen werden. Es ist sicher aufgefallen, dass, folgt man der Schrift, die buchstäbliche Graphie mit »il« beginnt und auch endet: »il« (neige au sole)il; während jedoch das erste »il« ein eigenständiges Wort ist, das erste des Satzes, ist das zweite »il« Teil des letzten Wortes (soleil). Der Wortabstand »il / neige«, im Gegensatz zu der Einbindung "soleil«, weist ganz klar darauf hin. Ein Gegensatz, den die phonè und das Hören mit dem Verschwinden der geschriebenen Reime in der Phonik bekräftigen: das "il « am Anfang ist phonetisch tatsächlich »il«, das »il« am Ende verschwindet in dem Diphthong »eil«. Wenn man sich die beiden ersten Proben von »il neige au soleil« genauer ansieht, stellt man fest, dass die expressive Kraft der graphischen Linie im graphischen Prozess besonders auf die Schrift hin drängt, unter der Stimme; denn der Dichter schreibt oder vielmehr der Zeichner-Dichter zeichnet das »IL« am Anfang und das »IL« am Ende identisch. Diese expressive Kraft des Traktus der Zeichnung verschiebt, in allen Bedeutungen des Wortes, den Verlauf der Schrift sowie die Linie der Stimme.

Die elf Variationen, die elf Neu-Schreibungen als Variationen desselben Satzes, haben keinen anderen Sinn und Zweck, als diese bereits in der ersten »Probe« präsente, aber hier noch unterschwellige expressive Kraft vollständig ans Licht zu bringen; um die Potenz des Visuellen, seine virtù, oder auch die Arbeit der Darstellbarkeit in Schrift und Wort, in der phonè, zu manifestieren. Damit können wir noch einmal auf das Thema der Wiederholung - der elf »Proben«, in der Sprache des Druckwesens, der elf Experimente, in der Sprache des Physiklabors, der elf Neu-Schreibungen, in der Sprache der Linguisten - zurückkommen, um in ihr die Gliederung der Serie und des Zyklus, des Variationsschemas und des Dispositivs der Transformation aufzuzeigen, die Emergenz einer poetischen (oder poietischen) Zeitlichkeit, einer Zeitlichkeit des Visuellen sowie des Textuellen, einer Zeitlichkeit der Zoographia (so bezeichneten die Griechen die Malerei), einer intensiven Zeitlichkeit, die weder sukzessiv ist - linear (jene der satzlichen, diskursiven Linie) - noch zirkulär - Erzeugerin Desselben (jene der Zeichnung, des mimetischen Bildes), die aber dort, an ein und demselben Ort und in ein und derselben Aussage den Sinn offenbart: die Epiphanie einer Kraft, das Sich-Zeigen einer Potenz, deren poetisches Emblem die Auflösung des ursprünglichen meteorologischen Oxymorons im solaren Triumph der letzten Probe wäre, in welcher der Schnee durch Überdeckung Bestandteil der Sonne wird.

Man kann also die elf Experimente als eine Serie von elf Sequenzen betrachten, die graphisch erzählen, wie sich am 10. Januar 1943 in Paris das Wetter zu diesem Meteor eines Schneefalls in der Sonne entwickelte, eine Entwicklung, die letztendlich auf die Auslöschung des Schnees vor oder in dem Licht und der Hitze der Sonne hinausläuft. Gleichzeitig aber macht die obstinate Wiederholung (in der Musik spricht man vom "Basso ostinato«) des immerselben Tempus des Präsens in »il neige« jede Okkurrenz von »il neige au soleil« zu einem jeweils neuen, unwiederholbaren, singulären Moment - hier zitiere ich Benveniste -, in dem sich das Zusammenfallen eines konkreten sprachlichen Äußerungsaktes mit dem in ihm mitgeteilten Geschehen verwirklicht: 
Jeder Äußerungsakt »il neige au soleil« verwirklicht ein absolutes, vollkommenes, selbstgenügendes Jetzt - den Augenblick reiner Anwesenheit. Weil aber diese Äußerung niedergeschrieben ist und weil ihrer Aussage, mit Tusche auf dem Medium ArchesPapier festgehalten, ihr eine Gegenwärtigkeit, eine "Aufrechterhaltung" verbürgt, bedeutet dieser schriftliche Vermerk gleichzeitig die unwiderrufliche Abwesenheit des Ereignisses, sein Nicht-mehr-Dasein, während diese Abwesenheit in dem graphischen Monument zugleich eine Transformation, eine "Erhöhung" erfährt: In dieser so erlangten Monumentalität liegt die Bedeutung des schriftlichen Vermerks eines Ortes und eines Datums (Paris, 10. Januar 1934) auf dem Papier, auf welchem, so ist es im Katalog zu lesen, mit Tusche geschrieben der zeitlich offene Satz steht, der nicht lautet: »il a neigé au soleil à Paris ce jour-là» [»in Paris hat es an jenem Tag in der Sonne geschneit«], sondern »il neige au soleil« [»es schneit in der Sonne«]. Somit enthüllen die elf kleinen Blätter, die elfmalige Wiederholung desselben Satzes, das Variationsschema, welches diese Wiederholung durchläuft und das Dispositiv der Transformation, welches die Variationen manifestieren, gemäß dieser present-tense, die sich auf das present-weather bezieht, eine present-time, die ich intensiv genannt habe, jene der poietischen Schöpfung, oder genauer gesagt, jene der Prägnanz des Visuellen im Textuellen, des Vordrängens des Blicks in die Lektüre der Buchstaben, des Hervortretens eines kosmischen Sonnenauges im weiß-grauen Fluss, im Schnee der Zeichen.

\section{BIBLIOGRAPHIE}

Arnauld, A. / Nicole, Pierre (1994 [1683]): Die Logik oder die Kunst des Denkens, aus dem Französischen von Christos Axelos, Darmstadt: Wiss. Buchgesellschaft.

Fumaroli, M (1984): „Muta eloquentia: la représentation de l'éloquence dans l'œuvre de Poussin«, Bulletin de l'histoire de l'art française.

Jacobson, R. (1970): »On the verbal art of William Blake and other poet-painters«, Linguistic Inquiry, 1, H. 1, S. 3-23.

Lee, R.W. (1942): »)Ut pictura poesis`: The humanistic theory of painting «, Art Bulletin, XXII, S. 197-269; auch in: Ders.: Ut Pictura Poesis. The Humanistic Theory of Painting, New York: Norton, 1967; Ders.: Ut Pictura Poesis. Humanisme et théorie de la peinture. XVe-XVIIIe siècles, frz. Übers. von Maurice Brock, Paris: Macula, 1991.

Picasso (1989): Écrits, herausgegeben und mit Anmerkungen versehen von M.L. Bernadac und Ch. Piot, Paris: R.M.N./Gallimard.

\section{NOTES}

1. "Dichtung sei wie Malerei; möge der Dichtung auch die Malerei ähnlich sein; jede der beiden Schwestern bezieht sich auf die andere als Rivalin. Abwechselnd alternieren ihre Namen, stumme 
Dichtung, sagt man von der einen, sprechende Malerei pflegt man die andere zu nennen.« [A.d.Ü.]

2. "Le Crayon qui parle, Picasso poète«, kuratiert von Marie-Laure Bernadac, Paris, Musée Picasso, 8. November 1989 bis 29. Januar 1990

3. Picasso (1989).

4. Jacobson (1970).

5. Vgl. zum Beispiel die Studie von Fumaroli (1984).

6. Lee (1942).

7. Word and Image, A Journal of Verbal/Visual Inquiry, London: Taylor and Francis. Die erste Ausgabe (Januar-März 1985) widmete sich Ut pictura poesis.

8. Es handelt sich um die Zeitschrift Artstudio Nr. 15 (Paris, Winter 1989).

9. Arnault / Nicole (1994), S. 25.

10. Arnault / Nicole (1994), S. 43.

INDEX

Schlüsselwörter : Schrift-Figur, ars poetica, Semiologie des Visuellen, Text und Bild, schöpferisches Experiment

Mots-clés : écriture-figure, ars poetica, sémiologie du visuel, texte et image, expérience créatrice

\section{AUTEURS}

\section{LOUIS MARIN}

Louis Marin (1931-1992), Kunstphilosoph. Nähere Informationen finden Sie hier. 